\title{
BMJ Open Associations of nicotine dependence and fat distribution in Chinese male adults: a cross-sectional study in Lanxi, China
}

Chen Wei, ${ }^{1,2}$ Sunyue Ye, ${ }^{\oplus 1,2}$ Jessica Ruolin Sheng, ${ }^{2,3}$ Xiaoguang Ma, ${ }^{1,2}$ Yuan $\mathrm{Ru},{ }^{1,2,4}$ Liang Zhang, ${ }^{5}$ Huilan Guo, ${ }^{1,2}$ Shankuan Zhu ${ }^{1,2}$

To cite: Wei C, Ye S, Sheng JR, et al. Associations of nicotine dependence and fat distribution in Chinese male adults: a cross-sectional study in Lanxi, China. BMJ Open 2019;9:e022465. doi:10.1136/ bmjopen-2018-022465

- Prepublication history for this paper is available online. To view these files, please visit the journal online (http://dx.doi org/10.1136/bmjopen-2018022465).

Received 21 February 2018 Revised 29 December 2018 Accepted 31 January 2019

Check for updates

(c) Author(s) (or their employer(s)) 2019. Re-use permitted under CC BY-NC. No commercial re-use. See rights and permissions. Published by BMJ.

${ }^{1}$ Department of Nutrition and Food Hygiene, School of Public Health, School of Medicine, Zhejiang University, Hangzhou, Zhejiang Province, China ${ }^{2}$ Chronic Disease Research Institute, School of Public Health, Zhejiang University, Hangzhou,

Zhejiang Province, China

${ }^{3}$ Department of Molecular Biology, Princeton University,

Princeton, New Jersey, USA

${ }^{4}$ Division of Nutritional Sciences, Cornell University, Ithaca, New York, USA

${ }^{5}$ Office of the Secretary, Lanxi Municipal Bureau of Health, Lanxi, Zhejiang Province, China

Correspondence to

Dr Shankuan Zhu;

zsk@zju.edu.cn

\section{ABSTRACT}

Introduction Previous studies have showed association between smoking and central fat distribution. However, the impact of smoking on whole body fat distribution, particularly peripheral fat distribution remains unclear. Methods Nicotine dependence was assessed in a total of 1264 male adults aged 18-80 years using the Fagerström Test for Nicotine Dependence (FTND). Smoking status was categorised as non-smokers, former and current smokers with very low, low/moderate, or high FTND scores. Body fat distribution was determined using the dual energy X-ray absorptiometry and anthropometric measurements. Multivariable linear regression models were applied to examine the adjusted associations between body fat distribution and smoking in all participants, and its association with FTND scores in the current smokers. Results Greater waist circumference (WC), waist-tohip ratio (WHR), waist-to-height ratio (WHtR), trunk fat percentage (\%TF), android fat percentage (\%AF) and android-to-gynoid fat mass ratio (AOI); but lower legs fat percentage (\%LegF), limb fat percentage (\%LimbF) and gynoid fat percentage (\%GF) were found in current smokers with high FTND scores compared with nonsmokers. In current smokers aged 60 years or older, FTND scores had positive associations with WC, WHR, WHtR, $\% \mathrm{TF}, \% \mathrm{AF}$ and $\mathrm{AOI}$, and negative associations with \%LegF, $\%$ LimbF and \%GF.

Conclusions Nicotine dependence was positively associated with central fat distribution and negatively associated with peripheral fat distribution in Chinese male adults, particularly in those older or heavy smokers, and these associations were independent from body mass index, which might be due to long exposure to smoking.

\section{INTRODUCTION}

Smoking is of a significant single cause of the preventable mortality in the world, ${ }^{1}$ and has been found to closely relate to body weight. ${ }^{2}{ }^{3}$ However, results from the previous studies have been complicated. When compared with non-smokers, current smokers tend to have lower body weight and body mass index (BMI); and smoking cessation can lead to weight gain. ${ }^{2-5}$ However,
Strengths and limitations of this study

- The present study examined the most thorough indices of fat distribution measured by a dual energy $X$-ray absorptiometry.

- Smoking status was evaluated using the Fagerström Test for Nicotine Dependence, which is more consistent with the biochemical markers of heaviness of smoking.

- This study does not establish any causal association between nicotine dependence and fat distribution for the cross-sectional study design.

- The energy intake was not taken into adjustment in this study.

neither body weight nor BMI can properly indicate central fat distribution, which is considered a discriminator of a cluster of chronic diseases. As current smokers tend to have greater waist circumference (WC), waistto-hip ratio (WHR) and higher prevalence of central obesity, ${ }^{267}$ it is important to consider body fat distribution in investigation the relationship between smoking and obesity. ${ }^{8-10}$

Most previous population-based studies used anthropometric indicators (eg, WC, WHR) to assess central fat distribution but these markers only indicate crude measurements of regional fat distribution. In order to capture a more precise association between smoking and body fat distribution, the dual energy X-ray absorptiometry (DXA) is utilised as a reliable measuring tool for both central and peripheral fat distribution. ${ }^{11-14}$

Self-reported exposure is the most commonly used method in a large scaled population-based study to evaluate the exposure to cigarette smoking. While the number of cigarettes smoked per day was the most frequently asked question in previous studies, it was not accurate for smoking assessment. ${ }^{1516}$ For example, saliva nicotine is a biochemical 
marker to assess the exposure to smoking, and over half of the variance of saliva nicotine depends on the factors other than the number of smoked cigarettes, including smoking depth and duration of inhalation, number of puffs per cigarette, and tapering of ventilation holes, and so on. ${ }^{15} 17$ The Fagerström Test for Nicotine Dependence (FTND) is a standard test to assess nicotine dependence of current smokers and has a high-level consistency with biochemical indices of heaviness of smoking. ${ }^{18}$ To our knowledge, the quantitative assessment of the direction and strength of nicotine dependence on body fat distribution has been poorly documented. Previous studies have not used FTND as an independent variable to quantitatively assess the association with fat distribution.

Therefore, this study aimed to investigate the association between fat distribution and smoking using the method of FTND in order to provide a complete profile of the associations of smoking with whole body fat distribution, including central and peripheral fat distribution.

\section{METHODS}

\section{Patient and public involvement}

Patients were not involved in the design or conduct of this study. The results on physical examination, body composition and blood test were integrated into a brief health report to each participant.

\section{Participants}

The study was conducted in 2015 in Lanxi, Zhejiang Province, China. Convenience sampling was performed to recruit participants. Extensive publicity campaigns included banner, poster, advertisement and radio. Invitation letters were delivered door-to-door by community leaders and health workers. Consent forms were collected from all the participants.

A total of 1264 male adults, aged 18-80 years old, were recruited in the study. All the participants joined this study voluntarily and gave consent for their health information to be used in the further studies. Participants who had missing information $(n=63)$ or did not take DXA scan $(n=30)$ were excluded, leading to an overall 1184 participants in the final analyses.

\section{Anthropometry and DXA}

Weight and standing height were measured in light clothing without shoes to the nearest $0.01 \mathrm{~kg}$ and $0.1 \mathrm{~cm}$, respectively. WC and hip circumference (HC) were measured using plastic flexible measuring tape to the nearest $0.1 \mathrm{~cm}$ (SECA 201, DE). WC was measured at the midpoint between the lower margin of the last palpable rib and the top of the iliac crest, ${ }^{19}$ and $\mathrm{HC}$ was defined as the circumference of the widest portion of the buttocks, with the tape parallel to the floor. ${ }^{19}$ All anthropometric values were measured three times and their mean was recorded. BMI, WHR and waist-to-height ratio (WHtR) were calculated and used as rough indicators of central obesity.
Total body fat mass (FM) and regional FM were measured utilising a whole-body scan with the DXA (GE-Lunar Prodigy, USA). For the android region, the upper boundary is above the pelvis cut by $20 \%$ of the distance between pelvis and femoral neck cuts and the lower boundary is at the pelvis cut. ${ }^{20} 21$ The gynoid region was defined as the upper boundary below the pelvis cut line by 1.5 times the height of the android region. The height is equal to two times the height of the android region, and the lateral boundaries are the outer leg cuts. ${ }^{2021}$ The android and gynoid FM indicate abdominal and hip fat distribution, respectively. ${ }^{20}{ }^{21}$ The per cent of body fat mass $(\% \mathrm{BF})$ was calculated as a body fat mass divided by a total body weight. The per cents of trunk fat mass (\% TF), arm fat mass (\%ArmF), leg fat mass $(\% \mathrm{LegF})$, android fat mass $(\% \mathrm{AF})$ and gynoid fat mass (\%GF) were calculated as trunk FM, android FM, gynoid FM, arm FM and leg FM divided by total body FM, respectively. The percent of limb fat mass (\% LimbF) was calculated as the sum of \% ArmF and \%LegF. Android-togynoid fat mass ratio (AOI) was calculated as android FM divided by gynoid FM.

All the measurements above were assessed and calculated by trained staff from the Zhejiang University, Hangzhou, China, following the standardised protocol. The DXA was calibrated before each use against a standard phantom provided by the manufacturer.

\section{Questionnaire survey}

All the participants were asked to complete a questionnaire that included demographic information, data on lifestyle and medical history. The questionnaire surveys were interviewed by trained personnel using an iPadbased direct data-entry system, with the function to avoid logical errors.

Marital status was categorised into two groups: married and others (unmarried, divorced or widowed). Education was classified into four levels: illiteracy, primary school, middle/high school and college or above. Alcohol use was categorised into two groups: regular $(\geq 1 /$ month currently) and others $(<1 /$ month currently or never/ former drinkers). Physical activity was assessed using a short version of the International Physical Activity Questionnaire. Based on the tertiled metabolic equivalence tasks, physical activity was classified into low, moderate and high groups.

A validated FTND (Chinese version) was applied to assess nicotine dependence, using scores of nicotine dependence ranging from 0 to 10 . Based on self-reported smoking habits and FTND scores, participants were categorised into five groups of smoking status: non-smokers, former and current smokers with very low, low/moderate and high FTND scores. Non-smokers were defined as those who never smoked or those who had smoked no more than 100 cigarettes in their lifetime. Former smokers were those who quitted smoking for 6 months or longer and did not smoke currently. Current smokers were those who had smoked 100 cigarettes or more in 
their lifetime and currently smoked daily or occasionally, and were categorised into three subgroups based on the FTND scores: very low (scores of 0-2), low and moderate (scores of 3-5) and high (scores of 6-10) nicotine dependence. $^{1822}$

\section{Physical examination}

After fasting for 12 hours overnight, venepuncture was conducted to measure participants' fasting plasma glucose, triglyceride, total cholesterol, high-density lipoprotein cholesterol, low-density lipoprotein cholesterol and blood uric acid. A certificated clinical laboratory analysed the blood samples. Blood pressure was measured at least twice by trained staff using an auscultation mercury sphygmomanometre. The average value of the two recorded measurements was used.

Results of the physical examination were combined with individual medical history to determine the presence of the four main metabolic conditions: dysarteriotony, pathoglycaemia, dyslipidaemia and hyperuricaemia. Participants with at least one of the four main metabolic conditions were defined as metabolically abnormal.

\section{Data analysis}

Continuous and categorical variables were presented as mean (SD) and numbers (proportions), respectively. Characteristics of participants were compared by analysis of variance for continuous variables or by $\chi^{2}$ test for categorical variables among the groups of smoking status.

Multivariable linear regression models were applied to compare fat distribution within the five groups of smoking status. The adjustment variables included age, BMI, marital status, education, alcohol use and metabolic abnormality. To further explore the associations between fat distribution and FTND scores (continuous variable), multivariable linear regression models were conducted among all current smokers. Due to the significant interactions between age and FTND scores for \% AF and AOI (data not shown), current smokers were stratified by age using 10-year intervals into four age groups and analysed separately. All the multivariable linear regression models were adjusted for age, BMI, marital status, education, physical activity, alcohol use and metabolic abnormality. A sensitivity analysis was performed by including participants with missing variables, encoded as mean for continuous and mode for categorical variables.

All the analyses were performed using SPSS V.20.0 (IBM, Armonk, New York, USA). $\mathrm{P}<0.05$ (two-tailed) was considered statistically significant.

\section{RESULTS}

\section{Basic characteristics of the participants}

The characteristics of the participants by smoking status are presented in table 1. Among the 1184 participants, the mean age, $\mathrm{BMI}$ and $\mathrm{BF} \%$ were $53.57 \pm 13.53$ years, $23.74 \pm 3.11 \mathrm{~kg} / \mathrm{m}^{2}$ and $23.62 \% \pm 6.29 \%$, respectively. There were 520 non-smokers, 488 current smokers and 176 former smokers in total participants. Among the current smokers, the numbers of the smokers with very low, low/ middle and high FTND were 241, 170 and 77, respectively. Significant differences in age, marital status, education, physical activity, height, HC, WHR, \%AF and AOI (all $\mathrm{p}<0.05$ ) were found among the five groups of smoking. Current smokers with high FTND scores had the greatest WHR, \%AF and AOI, and smallest HC.

\section{Association between smoking status and fat distribution}

The results of multivariable linear regression analysis by smoking status are presented in table 2. All regression models were adjusted for age, BMI, marital status, education, physical activity, alcohol use and metabolic abnormality. Compared with non-smokers, current smokers with high FTND scores had significantly higher values of WC, WHR, WHtR, \% TF, \% AF and AOI, and lower \% LegF, $\%$ LimbF and \%GF (all $\beta>0$ and $\mathrm{p}<0.05$ ). Meanwhile, current smokers with low/moderate FTND scores had significantly greater values of WHR $(\beta=0.08)$ and lower $\% \mathrm{BF}(\beta=-0.806)$ compared with non-smokers.

\section{Association between FTND scores and fat distribution}

The results of multivariable linear regression models on FTND scores and fat distribution in total current smokers and in each age group of current smokers are presented in table 3. After adjusting for age, BMI, marital status, education, physical activity, alcohol use and metabolic abnormality in the current smokers, the FTND scores only showed significantly positive associations with WC, WHR and WHtR among total current smokers (all $\beta>0$ and $\mathrm{p}<0.05$ ). When stratifying age to four layers, the FTND scores had significantly positive associations with WC, WHR, WHtR, \% TF, \%AF and AOI (all $\beta>0$ and $\mathrm{p}<0.05$ ), and significantly negative associations with \%LegF, $\%$ LimbF and \%GF (all $\beta<0$ and $\mathrm{p}<0.05$ ) in the group of 60 years or older current smokers. However, in the groups of 40-49 and 50-59 years old current smokers, FTND scores were positively associated with WC only $(\beta=0.268$ for $40-49$ years and $\beta=0.350$ for $50-59$ years).

Additionally, the results showed no significant differences between included and excluded participants with missing variables.

\section{DISCUSSION}

The present study found that current smokers with high FTND scores had greater values of WC, WHR, WHtR, $\% \mathrm{TF}, \% \mathrm{AF}$ and AOI, and lower \%LegF, \%LimbF and $\% \mathrm{GF}$ compared with non-smokers after controlling for age, BMI and other potential confounding factors. The current smokers, particularly 60 years or older current smokers, had FTND scores positively associated with central fat distribution and inversely associated with peripheral fat distribution.

Previous studies demonstrated that smoking was associated with central fat distribution using anthropometric indicators (eg, WC and WHR) alone. ${ }^{267}$ Consistent with 


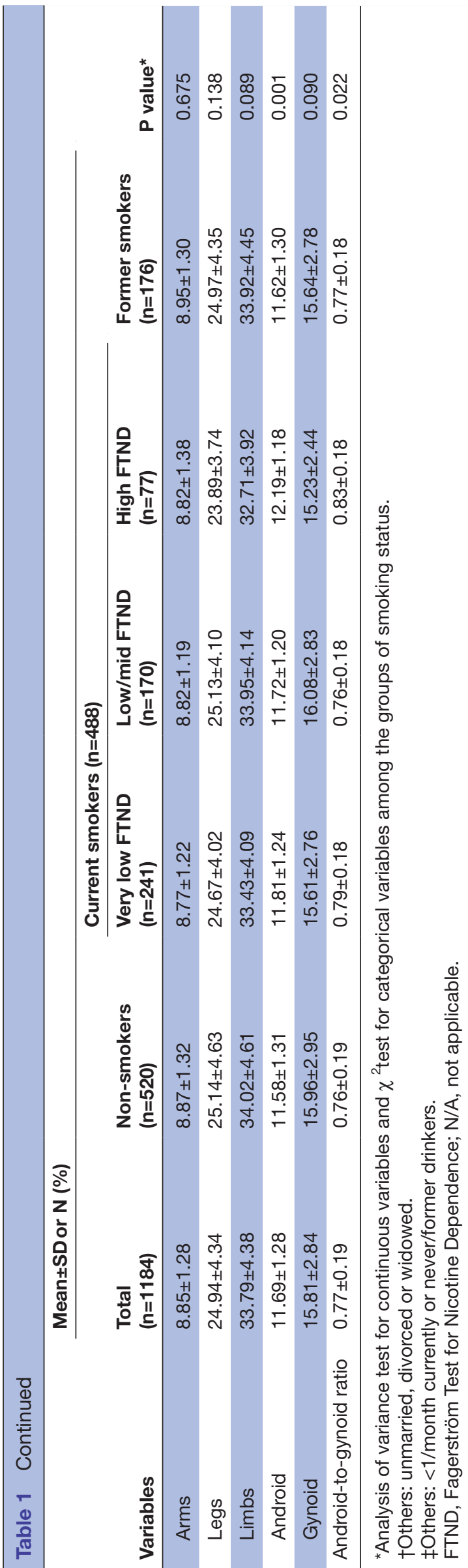

previous study, the association between smoking status and fat distribution in this study population showed that heavy smokers had more central fat than non-smokers. ${ }^{23}$ In the age stratification analyses, stronger associations between FTND scores and fat distribution were found in the current smokers with older age, which is consistent with previous studies. ${ }^{625}$

The effect of smoking on fat distribution is a chronic dose-response process. ${ }^{24}$ High nicotine dependence or age often implicate a heavier and longer exposure to smoking, thus the associations of smoking with fat distribution are likely to be observed in older or heavy smokers. As the number of younger current smokers was limited in this study, it might restrict the power to examine significant associations between smoking and fat distribution.

Compared with the previous population-based studies, this study examined the most thorough indices of fat distribution and provided a complete profile of the associations between smoking and whole-body fat distribution, including central and peripheral fat distribution. Using the DXA to measure fat distribution provided concise evidence to detect the changes in peripheral fat distribution in older or heavier smokers. Declined peripheral fat distribution might be the reason to weight loss and declined BMI in some smokers reported from the previous studies. Considering the single racial composition of the participants, the study's findings can be only applied to East Asians, and more multiracial evidence is in need for better generalisability. Given the similar BMI, Asians tend to have more central fat distribution than Caucasians do, ${ }^{26}$ suggesting that association between fat distribution and smoking might have an ethnical-specific importance for Asians. Additionally, the associations between smoking, muscle loss and sarcopenia were also reported elsewhere. ${ }^{27}$ However, studies on the association between nicotine dependence and skeletal muscle mass are limited and requires further evidence.

Despite that smoking is known to associate with body weight and fat distribution, the underlying biological mechanism remains unclear. It is mainly because nicotine acts at different organs and tissues in human body. Some noticeable hormones and internal secretions might be part of the physiological pathways between smoking and fat distribution. Cortisol concentrations are higher in smokers because of the stimulation of sympathetic nervous system activity induced by smoking. ${ }^{24}$ Experiment in vivo showed depot-specific response to high cortisol concentrations in the abdominal and gluteofemoral depots. ${ }^{28}$ Meanwhile, previous studies have found that high-dose nicotine induces higher cortisol concentrations in male smokers ${ }^{29}$ which might explain the mechanism of the associations between FTND score with central and peripheral fat distribution. Insulin resistance, a risk indicator for increased central adiposity, is considered to directly and independently relate to smoking. ${ }^{240}$ For male smokers, smoking may reduce testosterone concentrations, which is inversely associated with visceral fat mass. ${ }^{24}$ Moreover, adiponectin and leptin are associated with body fat 


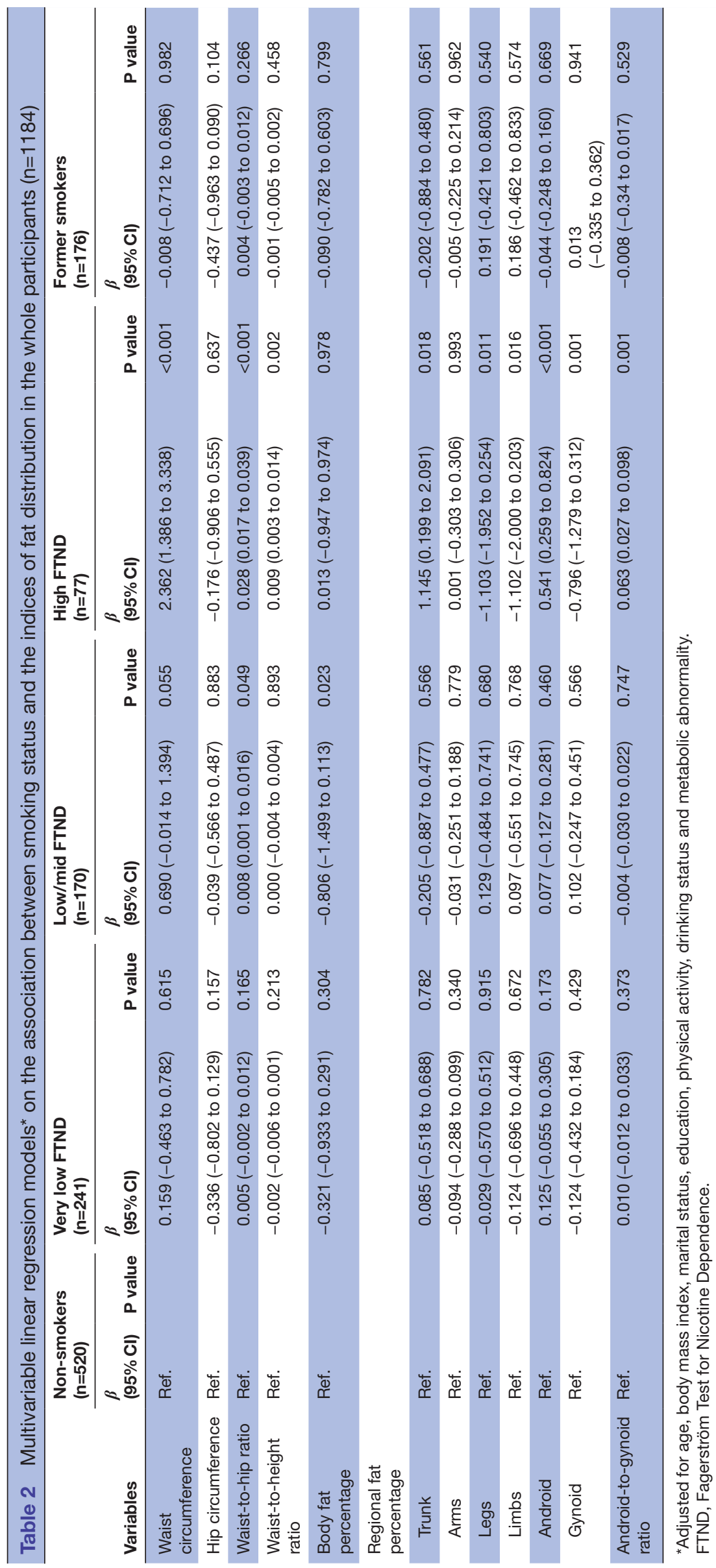




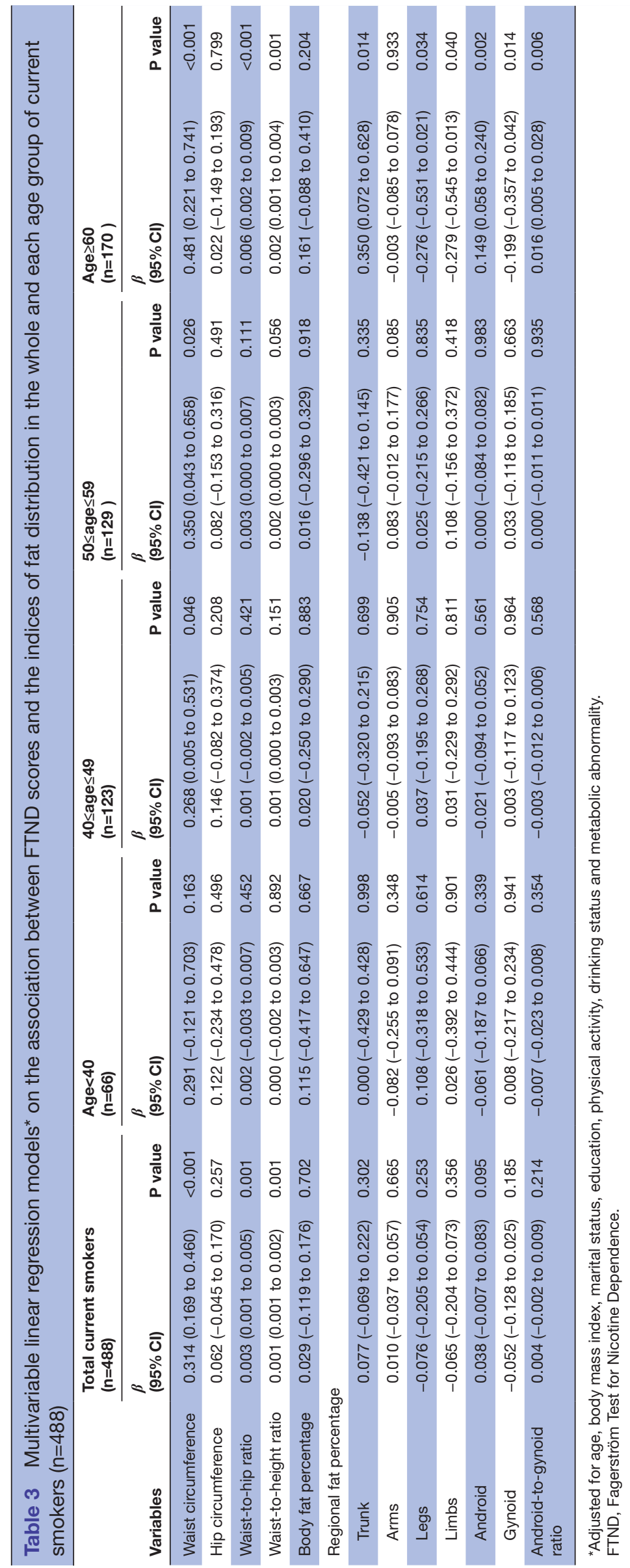


distribution and have different concentrations between smokers and non-smokers. ${ }^{31-33}$ In summary, a number of physiological factors affect associations between smoking and body fat distribution, but the network of these factors have not been well understood and more evidence is needed in the future.

There are several limitations in our study. First, the inherent limitation of the cross-sectional study is that it does not establish any temporal or causal association between nicotine dependence and fat distribution. The small sample size of subgroup might restrict the clinical importance of this study and further evidence from longitudinal studies is required. Second, potential self-report bias may exist in assessment of smoking exposure. However, FTND has a high level consistency with biochemical indices of heaviness of smoking, and is a reliable tool to evaluate nicotine dependence. ${ }^{18}$ Third, energy intake was not adjusted in the model, which might attenuate findings. However, it is well documented that nicotine suppressed appetite, reduced energy intake and increased energy expenditure,${ }^{34}{ }^{35}$ which inversely associated with abdominal obesity. ${ }^{36-38}$ We assumed that nicotine dependence was negatively associated with energy intake, and that adjustment for energy intake might not change the direction of findings. Meanwhile, we did not collect the type or dosage of alcohol use in this study, which should be improved in the future study.

\section{CONCLUSION}

This study shows a positive association between nicotine dependence and central fat distribution, and an inverse association between nicotine dependence and peripheral fat distribution. Both associations were independent of BMI in Chinese male adults, particularly for older or high FTND smokers.

Acknowledgements We acknowledge the Lanjiang Subdistrict Health Service Center and the Lanxi Municipal Bureau of Health. We are also grateful to community leaders and health workers for inviting eligible participators.

Contributors CW and SZ designed the study. SZ, CW, SY and LZ organised the fieldwork of this study. CW drafted the manuscript. SY, JRS, XM, YR, LZ and HG provided comments and revised the manuscript. CW and SZ had the responsibility for the integrity of the data analyses. All authors read and approved the final manuscript.

Funding This study was supported by the grant from the Cyrus Tang Foundation (419600-11102), with additional grants from the China Medical Board (CMB) Collaborating Program (15-216 and 12-108).

Competing interests None declared.

Patient consent for publication Obtained.

Ethics approval The ethics approval was provided by the Institutional Ethics Review Boards of School of Public Health Zhejiang University and the Lanxi Municipal Bureau of Health.

Provenance and peer review Not commissioned; externally peer reviewed. Data sharing statement Extra data is available by emailing Shankuan Zhu.

Open access This is an open access article distributed in accordance with the Creative Commons Attribution Non Commercial (CC BY-NC 4.0) license, which permits others to distribute, remix, adapt, build upon this work non-commercially, and license their derivative works on different terms, provided the original work is properly cited, appropriate credit is given, any changes made indicated, and the use is non-commercial. See: http://creativecommons.org/licenses/by-nc/4.0/.

\section{REFERENCES}

1. Proctor RN. The golden weed, America's most deadly drug. Science 2007;316:692-3.

2. Bamia C, Trichopoulou A, Lenas D, et al. Tobacco smoking in relation to body fat mass and distribution in a general population sample. Int $J$ Obes 2004;28:1091-6.

3. Munafò MR, Tilling K, Ben-Shlomo Y. Smoking status and body mass index: a longitudinal study. Nicotine Tob Res 2009;11:765-71.

4. Molarius A, Seidell JC, Kuulasmaa K, et al. Smoking and relative body weight: an international perspective from the WHO MONICA Project. J Epidemiol Community Health 1997;51:252-60.

5. Inoue K, Takeshima F, Kadota K, et al. Early effects of smoking cessation and weight gain on plasma adiponectin levels and insulin resistance. Intern Med 2011;50:707-12.

6. Akbartabartoori M, Lean ME, Hankey CR. Relationships between cigarette smoking, body size and body shape. Int $J$ Obes 2005;29:236-43.

7. Canoy D, Wareham N, Luben R, et al. Cigarette smoking and fat distribution in 21,828 british men and women: a population-based study. Obes Res 2005:13:1466-75.

8. Lee CM, Huxley RR, Wildman RP, et al. Indices of abdominal obesity are better discriminators of cardiovascular risk factors than BMI: a meta-analysis. J Clin Epidemiol 2008:61:646-53.

9. Ashwell M, Gunn P, Gibson S. Waist-to-height ratio is a better screening tool than waist circumference and BMI for adult cardiometabolic risk factors: systematic review and meta-analysis. Obes Rev 2012;13:275-86.

10. Nyamdorj R, Qiao Q, Lam TH, et al. BMl compared with central obesity indicators in relation to diabetes and hypertension in Asians. Obesity 2008;16:1622-35.

11. Park KS, Lee DH, Lee J, et al. Comparison between two methods of bioelectrical impedance analyses for accuracy in measuring abdominal visceral fat area. J Diabetes Complications 2016;30:343-9.

12. Park YW, Heymsfield SB, Gallagher D. Are dual-energy X-ray absorptiometry regional estimates associated with visceral adipose tissue mass? Int J Obes Relat Metab Disord 2002;26:978-83.

13. Ling $\mathrm{CH}$, de Craen AJ, Slagboom PE, et al. Accuracy of direct segmental multi-frequency bioimpedance analysis in the assessment of total body and segmental body composition in middle-aged adult population. Clin Nutr 2011;30:610-5.

14. Glüer CC. 30years of DXA technology innovations. Bone 2017;104:7-12.

15. Etter JF, Perneger TV. Measurement of self reported active exposure to cigarette smoke. J Epidemiol Community Health 2001;55:674-80.

16. Connor Gorber S, Schofield-Hurwitz S, Hardt J, et al. The accuracy of self-reported smoking: a systematic review of the relationship between self-reported and cotinine-assessed smoking status. Nicotine Tob Res 2009;11:12-24.

17. Herning RI, Jones RT, Benowitz NL, et al. How a cigarette is smoked determines blood nicotine levels. Clin Pharmacol Ther 1983;33:84-90

18. Heatherton TF, Kozlowski LT, Frecker RC, et al. The fagerström test for nicotine dependence: a revision of the fagerström tolerance questionnaire. Br J Addict 1991;86:1119-27.

19. World Health Organization. Waist circumference and waist-hip ratio: report of a WHO expert consultation: World Health Organization Report, 2011.

20. Fu X, Ma X, Lu H, et al. Associations of fat mass and fat distribution with bone mineral density in pre- and postmenopausal Chinese women. Osteoporos Int 2011;22:113-9.

21. Samsell L, Regier M, Walton C, et al. Importance of android/gynoid fat ratio in predicting metabolic and cardiovascular disease risk in normal weight as well as overweight and obese children. $J$ Obes 2014;2014:1-7.

22. Fagerström KO, Kunze M, Schoberberger R, et al. Nicotine dependence versus smoking prevalence: comparisons among countries and categories of smokers. Tob Control 1996;5:52-6.

23. Clair C, Chiolero A, Faeh D, et al. Dose-dependent positive association between cigarette smoking, abdominal obesity and body fat: cross-sectional data from a population-based survey. BMC Public Health 2011;11:23.

24. Chiolero A, Faeh D, Paccaud F, et al. Consequences of smoking for body weight, body fat distribution, and insulin resistance. Am J Clin Nutr 2008;87:801-9. 
25. Kuk JL, Saunders TJ, Davidson LE, et al. Age-related changes in total and regional fat distribution. Ageing Res Rev 2009;8:339-48.

26. He W, Zhang S, Song A, et al. Greater abdominal fat accumulation is associated with higher metabolic risk in Chinese than in white people: an ethnicity study. PLoS One 2013;8:e58688.

27. Szulc P, Duboeuf F, Marchand F, et al. Hormonal and lifestyle determinants of appendicular skeletal muscle mass in men: the MINOS study. Am J Clin Nutr 2004;80:496-503.

28. Manolopoulos $\mathrm{K}$, Thornhill $\mathrm{H}$, Thomas J, et al. Association between hypercortisolaemia and adipose tissue blood flow in vivo. Lancet 2015;385:S63.

29. Wilkins JN, Carlson HE, Van Vunakis $\mathrm{H}$, et al. Nicotine from cigarette smoking increases circulating levels of cortisol, growth hormone, and prolactin in male chronic smokers. Psychopharmacology 1982;78:305-8.

30. Patel P, Abate N. Body fat distribution and insulin resistance. Nutrients 2013;5:2019-27.

31. Hilawe EH, Yatsuya $\mathrm{H}$, Li Y, et al. Smoking and diabetes: is the association mediated by adiponectin, leptin, or C-reactive protein? J Epidemiol 2015;25:99-109.
32. Guenther M, James R, Marks J, et al. Adiposity distribution influences circulating adiponectin levels. Transl Res 2014;164:270-7.

33. Takahashi M, Funahashi T, Shimomura I, et al. Plasma leptin levels and body fat distribution. Horm Metab Res 1996;28:751-2.

34. Audrain-McGovern J, Benowitz NL. Cigarette smoking, nicotine, and body weight. Clin Pharmacol Ther 2011;90:164-8.

35. Stojakovic A, Espinosa EP, Farhad OT, et al. Effects of nicotine on homeostatic and hedonic components of food intake. J Endocrinol 2017;235:R13-R31.

36. Singh RB, Niaz MA, Bishnoi l, et al. Effect of low energy diet and weight loss on major risk factors, central obesity and associated disturbances in patients with essential hypertension. J Hum Hypertens 1995;9:355-62.

37. Janssen I, Fortier A, Hudson R, et al. Effects of an energy-restrictive diet with or without exercise on abdominal fat, intermuscular fat, and metabolic risk factors in obese women. Diabetes Care 2002;25:431-8.

38. Colles SL, Dixon JB, Marks P, et al. Preoperative weight loss with a very-low-energy diet: quantitation of changes in liver and abdominal fat by serial imaging. Am J Clin Nutr 2006;84:304-11. 